Original Contribution

\title{
PREGNANCY RELATED PLASMA PROTEIN-A AND PREMATURE BIRTH
}

\author{
Iv. Todorovi*, N. Tododrovi', M. Angelova ${ }^{1}$, K. Peeva ${ }^{2}$ \\ ${ }^{1}$ Department of Obstetrics\&Gynecology, Faculty of Medicine, Trakia University, \\ Stara Zagora, Bulgaria \\ ${ }^{2}$ Department of Social Medicine and Healthcare Management, Faculty of Medicine, \\ Trakia University, Stara Zagora, Bulgaria
}

\begin{abstract}
Introduction: Children born prematurely are at higher risk of mortality, morbidity, and impaired motor and cognitive development in childhood than prematurely born babies.

Aim: To establish the relationship between the corresponding levels of pregnancy-related plasma protein-A (PAPP-A) and the frequency of premature birth.

Materials and methods: The study is prospective. The data was collected through monitoring patients through a questionnaire and sonographic examination at 11-13 gestational weeks. The study excluded all known risk factors for preterm birth, such as previous preterm births, pregnant women with gestational diabetes, preeclampsia, hypertension, placenta previa, hydramnion, multiple pregnancies, smoking, structural and chromosomal abnormalities of the fetus and planned preterm birth. The data from the measured values of PAPP-A and the frequency of premature birth in 636 pregnant women were analyzed.

Conclusions: PAPP-A levels are a statistically significant factor for preterm birth. It is expected with a 95\% probability in the population with PAPP-A values below 0,515 that the cases with premature birth will be from 7 to 14 times more.

Pregnant women with PAPP-A level less than 10th per cent are significantly associated with an increased risk of preterm birth.
\end{abstract}

Key words: First-trimester screening, Pregnancy-associated plasma protein-A (PAPP-A), Preterm birth, Pregnancy outcomes.

\section{INTRODUCTION}

In daily practice, the study of pregnancyrelated plasma protein-A (PAPP-A), in combination with the $\beta$-fraction of human chorionic gonadotropin $(\beta-\mathrm{hCG})$ and ultrasound measurement of NT between 11 and 13 years plus 6 days of pregnancy is widely used as a biochemical screening to calculate the risk of Trisomy 21 and other aneuploidies (1). The marker PAPP-A is produced in the syncytiotrophoblast of the placenta and is a major source of circulating plasma protein-A in the maternal serum (2). It helps to create a free insulin-like factor (IGF), which plays an important role in the trophoblast invasion in

*Correspondence to: Ivan Todorov Todorov, Stara Zagora 6000, 171 "General Stoletov" Street, Email: mdtyt77@abv.bg, Тел.+359889298787 uterine decidua (3). Low levels of PAPP-A in the maternal serum lead to low levels of IGF, which can lead to abnormal trophoblast development and hence to various adverse complications of pregnancy, such as abortion, fetal developmental delay (IUGR), more frequent development of preeclampsia (PE), premature birth and oligohydramnios. Because PAPP-A serum marker screening is used worldwide to rule out fetal aneuploidy, low levels have been found to be associated with premature birth and other adverse effects of pregnancy $(4,5)$. Children born prematurely are at higher risk of mortality, morbidity and impaired motor and mental development in childhood than those born at term.

There are currently some differences in the definition of preterm birth. According to the Ministry of Health, a premature (untimely, 
TODOROV IV., et al.

embryonic) birth is the birth of a fetus with a birth weight of 800-2499 grams, including/or at gestational age of 26 and/or less than 37 completed gestational weeks, regardless of whether it is alive or dead. Premature birth is also established if the body weight of the fetus is below 800 grams and the age is below 26 gestation weeks, provided that it was born alive and lived for at least 3 days.

The most widely used and accepted definition of preterm birth is that of the World Health Organization (WHO), which defines preterm birth as any birth before 37 full weeks of pregnancy or less than 259 days from the first day of a woman's last menstrual period (LMP). This is further divided on the basis of the gestational age (GA):

- extremely premature (up to 28 weeks);

- very premature (28 to 32 weeks);

- moderate or late premature birth (32 to 37 weeks of pregnancy).

\section{Epidemiology}

The limitation of the WHO definition is that there is no boundary between miscarriage and the birth of a viable fetus, which complicates the assessment of premature birth. According to WHO data from 2015, about 10-15\% of premature babies are born annually (15 million premature babies), and almost 1 million of them die due to complications related to early birth.

Data from 184 countries on all continents show that the percentage of premature births varies from 5 to $18 \%$ and the poorer a country is, the more premature births. Data for Europe from 2013 are for a level of prematurity from $\mathbf{5 . 5}$ to $11.5 \%(6)$.

According to the data from the Association of Neonatology, the average percentage of premature births in Bulgaria in recent years ranges between 9.5 and $10.5 \%$. In addition, in most previous publications, the presence of other predisposing factors for preterm birth is not strictly controlled. We conducted this study to determine the strength of the relationship between PAPP-A levels and the development of "ideopathic preterm birth" (preterm birth without other predisposing factors and causes) using our own reference range.

\section{Objectives}

To establish the values of PAPP-A leading to premature birth.
To establish the strength and the relationship between the corresponding levels of pregnancy-related plasma protein-A (PAPP-A) and the frequency of premature birth.

\section{MATERIALS AND METHODS}

The study covered 636 consecutive singleton pregnancies in women undergoing screening in the first trimester. The duration of pregnancy during the blood test was calculated on the date of the last regular menstruation and was confirmed by ultrasound examination of the parietal-sacral length (CRL). This study excludes all known risk factors for preterm birth, such as previous preterm births, gestational diabetes, preeclampsia, hypertension, placenta previa, hydramnion, multiple pregnancy, smoking, structural and chromosomal abnormalities of the fetus and planned preterm birth.

Patients have given informed consent to the use of their data. The venous blood was tested in a laboratory using the DELFIA Xpress system (Perkin Elmer, Waltham, MA, USA). PAPP-A values were modified as MoMs (multiples of mediane).

All pregnant women with a PAPP-A level below $0.5 \mathrm{MoM}$ in the first trimester during the biochemical screening were selected in a separate target group, while women with normal levels at the same gestational age were used as a control group. The selected group of pregnant women with PAPP-A levels below $0.5 \mathrm{MoM}$ is based on reports in the literature of a more common adverse pregnancy outcome at these levels $(4,5,7)$. Information on the course of pregnancy and childbirth is obtained from the protocols of women's consultations (LC) and maternity wards.

Statistical analysis was performed using IBM SPSS version 25.0.

Disease with a $95 \%$ confidence interval was calculated.

ROC analysis was used and a ROC curve was constructed to determine the diagnostic efficacy of low PAPP-A levels in predicting birth problems. ROC analysis determined the cut-off value, with the maximum amount of sensitivity and specificity. Logistic regression was used, the odds ratio was calculated to assess the prognosis of problems in the course of pregnancy in cases with low PAPP-A with 95\% confidence intervals. Significance level P $<0.05$ was established. 


\section{RESULTS}

A total of 636 pregnant women were studied for a period of 5 years - from 2014 to 2019 . Of
TODOROV IV., et al.

the 636 women who gave birth, in 104 pregnant women $(16 \%)$ the PAPP-A values were below 0.515 MoM. (Figure 1).

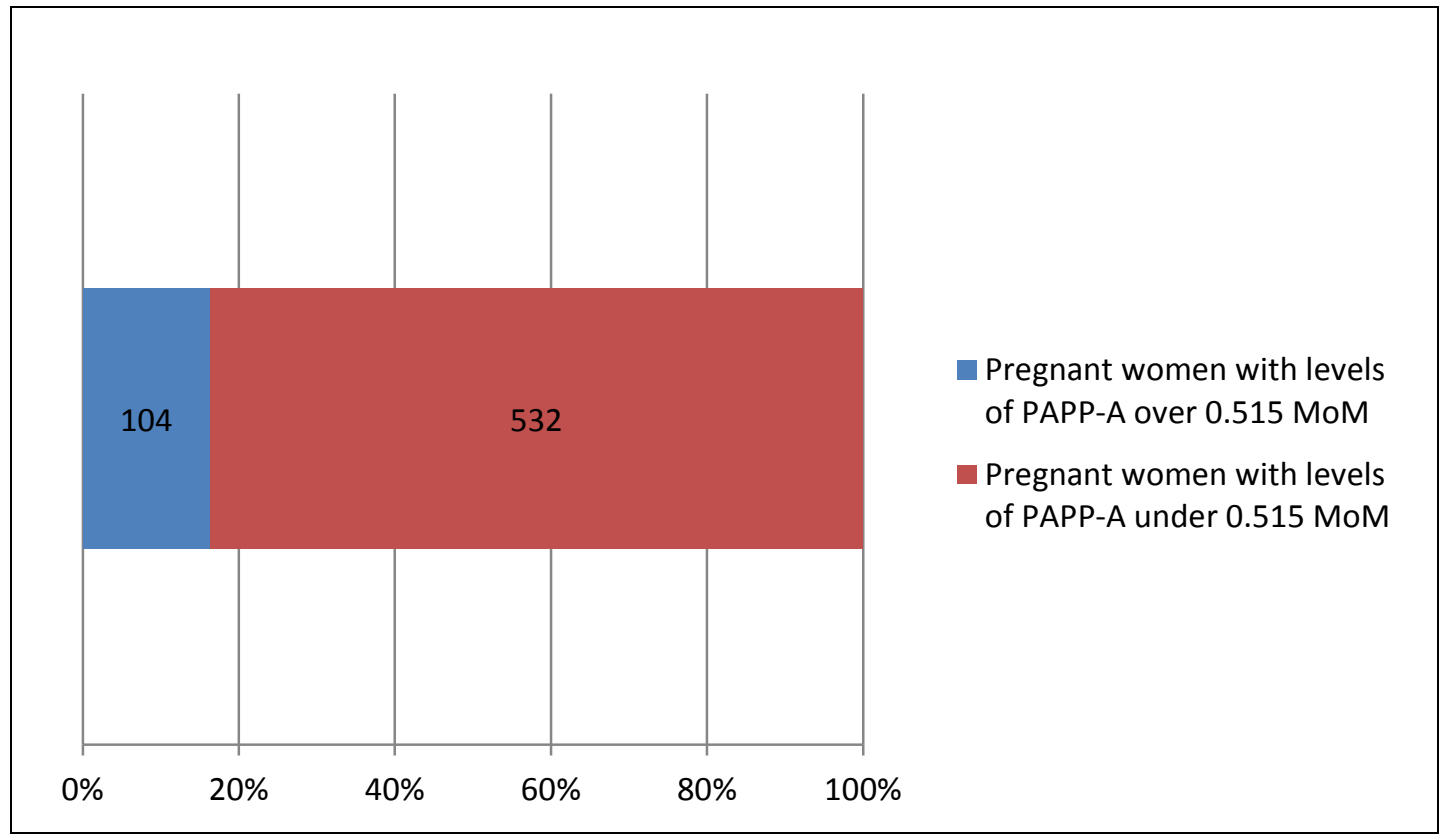

Figure 1. Frequency of pregnant women with low levels of PAPP-A

Out of 104 pregnant women with low levels of PAPP-A (MoM <0.515), 39 women had an unfavorable pregnancy outcome, such as: pregnancy loss, premature birth, preeclampsia, hypertension and retarded fetus birth.
In the control group of pregnant women with normal PAPP-A levels (MoM> 0.515), who were 532 women, these complications occurred in 39 pregnant women (Table 1).

Table 1. Complicated pregnancy and childbirth at low PAPP-A levels and in the control group.

\begin{tabular}{|c|c|c|c|}
\hline \multicolumn{4}{|c|}{ Total number of pregnant women- 636 } \\
\hline $\begin{array}{c}\text { With level of PAPP-A<0,515 MoM - 104 pregnant } \\
\text { women }\end{array}$ & \multicolumn{2}{c|}{$\begin{array}{c}\text { With level of PAPP-A }>0,515 \text { MoM - 532 pregnant } \\
\text { women }\end{array}$} \\
\hline $\begin{array}{c}\text { Complicated pregnancy } \\
\text { and birth }\end{array}$ & $\begin{array}{c}\text { Normal pregnancy and } \\
\text { birth }\end{array}$ & $\begin{array}{c}\text { Complicated pregnancy } \\
\text { and birth }\end{array}$ & $\begin{array}{c}\text { Normal pregnancy and } \\
\text { birth }\end{array}$ \\
\hline 46 & 58 & 39 & 493 \\
\hline $\mathbf{4 4 , 2 3 \%}$ & $55,77 \%$ & $\mathbf{7 , 3 3 \%}$ & $92,73 \%$ \\
\hline
\end{tabular}

In the target group (PAPP-A $<0.515 \mathrm{MoM}$ ) in $55.77 \%$ of the cases healthy full-term babies were born and in $44.23 \%$ there were complications of pregnancy and childbirth, such as premature birth, hypertensive diseases - preeclampsia or gestational hypertension (PE or $\mathrm{PIH})$, young children of gestational age (SGA), fetal loss. In the control group, 92.73\% of healthy full-term babies were born and all such complications were $7.33 \%$. These differences were statistically significant ( $P$ $<0.0001)$.

The distribution of the various complications of pregnancy in pregnant women with low levels of PAPP-A and in the control group are illustrated in Figure 2 and Figure 3.

The two figures show that the largest share of complications of pregnancy are: premature birth, PE and PIH, SGA and others (trisomy 21; fetal loss after 12 weeks of gestation).

There are 627 pregnant women with live births. Of these, 29 children were born prematurely $(4.62 \%)$. Of the pregnant women with preterm birth, 12 were with PAPP-A $<0.5$ MoM and 17 with PAPP-A> 0.5 MoM. The results of the percentage distribution of preterm infants are shown in Table 2. 


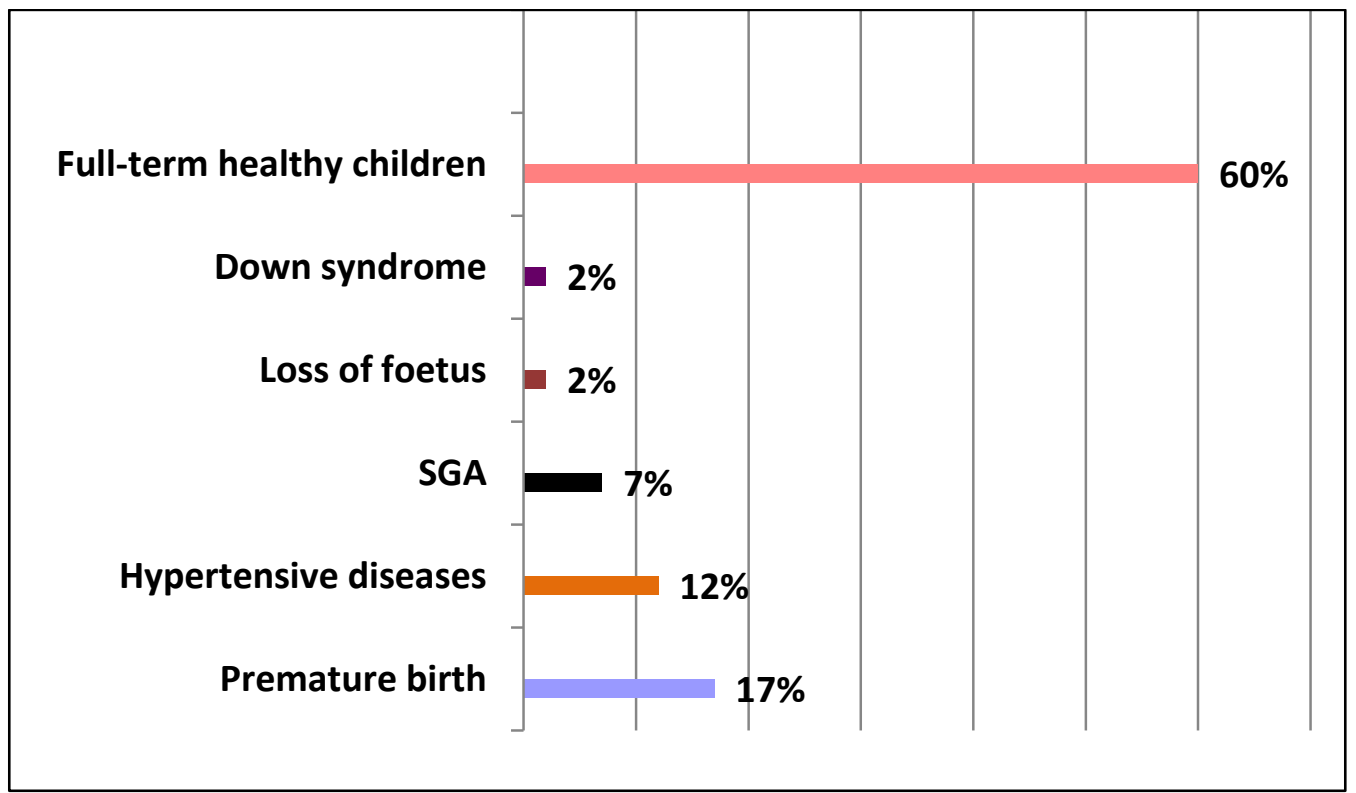

Figure 2. Pregnancy complications in pregnant women with PAPP-A level $\leq 0.5 \mathrm{MoM}$

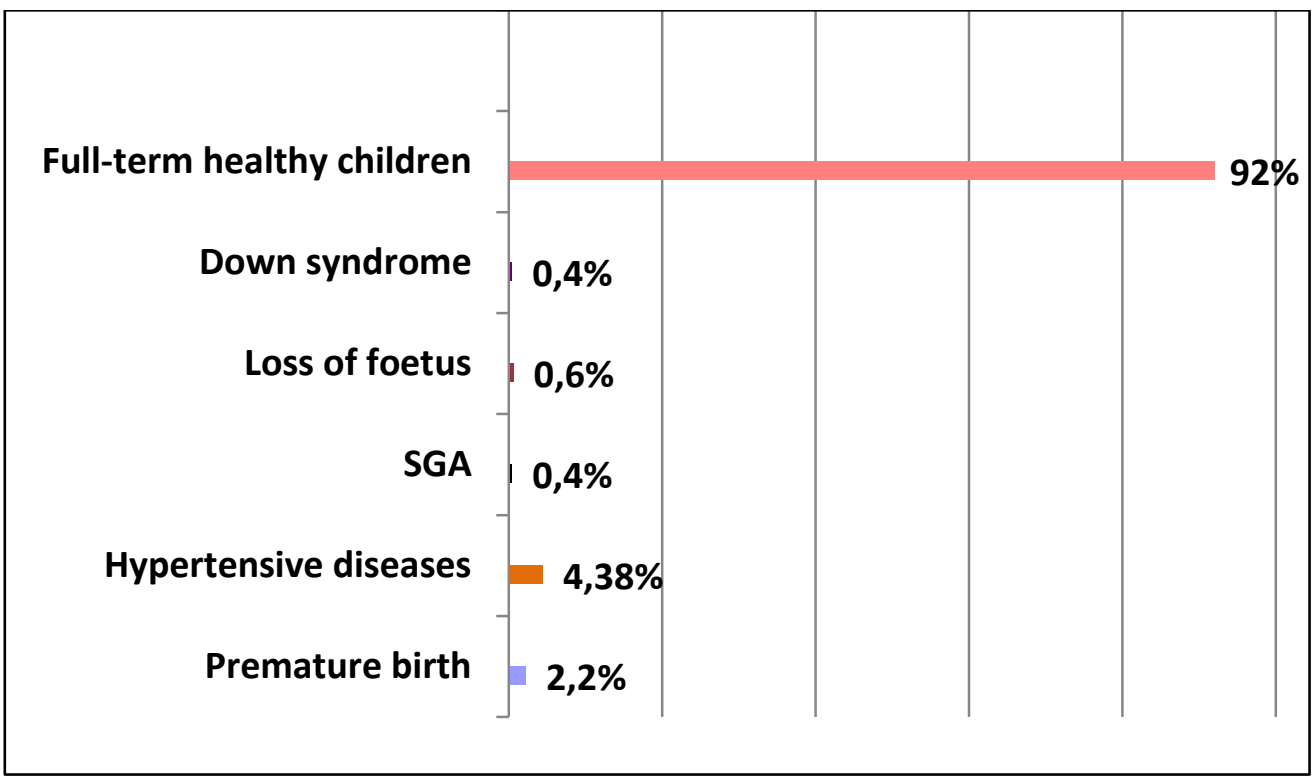

Figure 3. Complications of pregnancy in the control group - PAPP-A over 0.5MoM

Table 2. Distribution of premature births according to the level of PAPP-A

\begin{tabular}{|c|c|}
\hline PAPP-A $<0,515 \mathrm{MoM}, \mathrm{N}=99$ & PAPP-A $>0,515$ MoM, $\mathbf{N}=528$ \\
\hline $\begin{array}{c}\text { Number of pregnant women with premature } \\
\text { birth 12 }\end{array}$ & $\begin{array}{c}\text { Number of pregnant women with premature } \\
\text { birth } 17\end{array}$ \\
\hline $\mathbf{1 2 , 1 2 \%}$ & $\mathbf{3 , 2 1 \%}$ \\
\hline
\end{tabular}

The table shows that the level of PAPP-A is definitely different for the two groups and this difference is statistically significant $(\mathrm{P}$ $<0.0001)$. PAPP-A levels are a statistically significant factor for preterm birth.
In many studies, different authors report different cut-off values for the PAPP-A level expressed in MoM. Most opinions are grouped around a cut-off below 0.4 MoM. $(8,9)$ From the ROC analysis of our data, the breakpoint, cut-off is 0.515 MoM (Figure 4). 


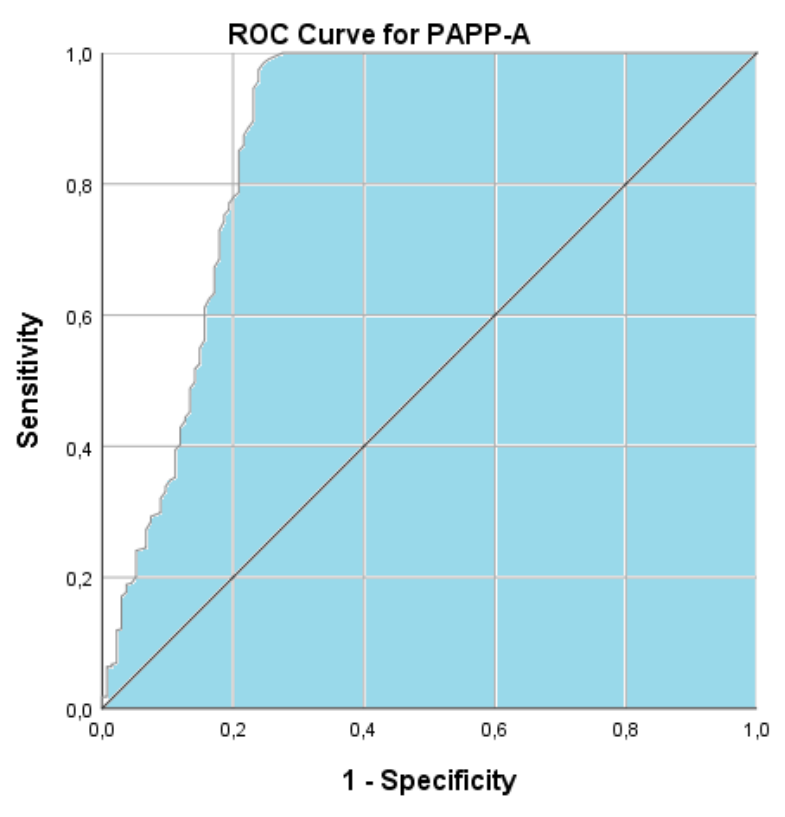

Diagonal segments are produced by ties.

Figure 4. Predicted PAPP-A levels <0.515 for complications during pregnancy (the area below the ROC curve 0.870 ).

Because the study is a slice, it is possible to calculate the incidence of "preeclampsia" and it is $5.7 \%$ with a $95 \%$ confidence interval (3.9; $7.5 \%)$.

It is expected with a $95 \%$ probability in the population with PAPP-A values below 0.515 , the cases with premature birth to be from 7 to 14 times more.

\section{DISCUSSION}

Pregnant women with PAPP-A levels below the 10th percentile are associated with a significantly increased risk of preterm birth (4, $5,7)$. According to some authors, the study of the level of serum PAPP-A independently performs poorly as a screening test for premature birth. The prognostic value is improved by the inclusion of additional factors (10-12).

According to the results of the study presented here, its significance is greater if only the cases of "idiopathic premature birth" are taken into account.

\section{CONCLUSION}

This study excludes all known risk factors for preterm birth and the results give us reason to conclude that the level of PAPP-A is an independent risk factor for ideopathic premature birth.

Measurement of the sonographic length of the cervix (CL) is known to be the most useful tool for assessing the risk of spontaneous preterm birth in asymptomatic women in the middle of the pregnancy (13).

Cases of low levels of PAPP-A in the first trimester may focus the attention on tracking the relevant pregnant women and measuring the length of the cervix. Other biochemical and genetic factors (alpha-fetoproteins, insulin-like growth factor IGFBP-1, progesterone receptors, etc.) may be studied in pregnant women with low levels of PAPP-A and a shortened cervix.

This can help clinicians monitor and prevent preterm birth in cases with low levels of PAPP-A, regardless of other risk factors. Although the main purpose of using firsttrimester serum indicators is to screen for aneuploidies, they are informative and are likely to be very useful in predicting preterm birth.

\section{REFERENCES}

1. Nikolaides K. N. Nuhal translucency and other first trimester sonographic markers of chromosomal abnormalities. Am. J. Obstet Gynecol. 2004; 191(1):45-67.

2. Handschuh K. Low PAPP-A: what are the clinical implications? Placenta. 2006; 27:127-134.

3. Smith GC. Pregnancy associated plasma protein $A$ and alpha-fetoprotein and 
prediction of adverse perinatal outcome. Obstet Gynecol. 2006; 107(1);161-6.

4. Jyoti Malik. Pregnancy-associated plasma protein A - a level in first trimester and its impact on pregnancy outcome. International Journal of Reproduction, Contraception, Obstetrics and Gynecology. 2016 A;5(8):2680-2683

5. Pummara P. Association of first-trimester pregnancy-associated plasma protein A levels and idiopathic preterm delivery: A population-based screening study. Taiwan $J$ Obstet Gynecol. 2016;55(1):72-5.

6. Zeitlin J. Preterm birth time trends in Europe: a study of 19 countries. International Jornal of Obstetrics and Gynecology. 2013; 120(11):1356-1365

7. Krantz, David. Association of extreme firsttrimester free human chorionic gonadotropin-beta, pregnancy-associated plasma protein A, and nuchal translucency with intrauterine growth restriction and other adverse pregnancy outcomes. Am J Obstet Gynecol. 2004; 191(4):1452-8.

8. Vesna Livrinova. Obstetric Outcome in Pregnant Patients with Low Level of
TODOROV IV., et al. Pregnancy-Associated Plasma Protein A in First Trimester. Macedonian Journal of Medical Sciences. 2018. Jun 20; 6(6):10281031.

9. Imcha M, Egbase E, Ross G. Outcome of pregnancy with low PAPP-A. Archives of disease in childhood. Arch Dis Child Fetal Neonatal Ed. 2014; 99:1359-2998

10.Proctor LK, Toal M, Keating S. Placental size and the prediction of severe early onset intrauterine growth restriction in women with low pregnancy associated plasma protein A. Ultrasounds Obstet Gynecol. 2009; 34(3):274-82.

11.Gagon A, Wilson RD, Audibert F. Obstetric complications associated with abnormal maternal serum marker analytes, $J$ Obset Gynecol. 2008;30(10):918-49.

12.Mithil Patil, T. M. Panchanadikar, Girija Wagh. Variation of Papp-A Level in the First Trimester of Pregnancy and Its Clinical Outcome. The Journal of Obstetrics and Gynecology of India. 2014; 64:116-119 